Original Research Article

\title{
A prospective randomised open labelled comparative study of anti inflammatory effects of topical $5 \%$ benzoyl peroxide gel vs topical $4 \%$ nicotinamide gel for grade I-II acne in a tertiary care hospital
}

\author{
Ashok Kumar S. N. ${ }^{1 *}$, Madan Mohan N. T. ${ }^{2}$, Shwetha H. ${ }^{1}$, Shanmukananda P. ${ }^{1}$, \\ Veena D. R. ${ }^{1}$, Upasana Dube ${ }^{1}$
}

\begin{abstract}
${ }^{1}$ Department of Pharmacology, ${ }^{2}$ Department of Dermatology, Dr. B R Ambedkar Medical College, Bangalore, Karnataka, India
\end{abstract}

Received: 08 March 2018

Revised: 18 March 2018

Accepted: 09 April 2018

\section{*Correspondence to:}

Dr. Ashok Kumar S. N., Email: drashokpharmacology @ gmail.com

Copyright: (C) the author(s), publisher and licensee Medip Academy. This is an openaccess article distributed under the terms of the Creative Commons Attribution NonCommercial License, which permits unrestricted noncommercial use, distribution, and reproduction in any medium, provided the original work is properly cited.

\begin{abstract}
Background: Acne vulgaris is a dermatological disorder characterised by formation of comedones and inflammatory lesions. The treatment of acne basically involves reduction of lesions. Benzoyl peroxide, in concentrations of $5 \%, 10 \%$, and $20 \%$, has been used effectively in the treatment of acne for more than 20 years. Nicotinamide/ Niacinamide is a newly-approved anti-acne drug with a potent anti-inflammatory effect. The present study assessed the efficacy of $5 \%$ Benzoyl peroxide gel in comparison to $4 \%$ Nicotinamide gel for topical treatment of mild to moderate acne vulgaris.

Methods: In this study, the patients with mild to moderate acne vulgaris with inflammation were divided into two groups, group I was treated with topical 5\% Benzoyl peroxide gel whereas topical Nicotinamide gel was given to the group II. Assessment of efficacy was done by total lesion counting according acne global severity index, the results were compared at the end of 2 weeks and 4 weeks with the baseline values.

Results: At the end of this study, it was found that the reduction of inflammatory and total percentage of decrease in counts of lesions from baseline were highly significant in both the groups $(\mathrm{p}<0.001)$, between the groups, differences were statistically significant $(\mathrm{p}<0.001)$, therefore $5 \%$ Benzoyl peroxide gel has better efficacy than $4 \%$ Nicotinamide gel.
\end{abstract}

Conclusions: Benzoyl peroxide is more efficacious than $4 \%$ Nicotinamide gel in mild to moderate acne.

Keywords: Acne vulgaris, Comedones, Inflammatory

\section{INTRODUCTION}

Acne, a chronic inflammatory disease of the pilosebaceous units of the face, neck, chest, and back, is the most common skin disorder occurring universally, with an estimated prevalence of $70-87 \%$. $^{1}$

It is a pleomorphic disorder characterized by both noninflammatory (comedones) and inflammatory (papules, pustules, nodules) lesions. Grading of acne is mandatory to determine the appropriate therapeutic strategy. Effective treatment can dramatically improve a person's quality of life. ${ }^{2}$

Benzoyl peroxide is frequently used as a first-line therapy for mild to moderate acne. Benzoyl peroxide acts through oxidation and formation of free radicals causing a reduction of $\mathrm{P}$. acnes. This mechanism helps to prevent an induction of resistance in P. acnes often observed during long term acne treatment with antibiotics. ${ }^{3}$ 
Niacinamide is a newly-approved anti-acne drug with a potent anti-inflammatory effect. Reduction of inflammation is a major mechanism of anti-acne treatment. More recent studies have noted that topical niacinamide is extremely well tolerated by facial skin that this agent provides several beneficial effects in reducing sebum production. ${ }^{4,5}$

The present study was done to compare the efficacy of 5\% Benzoyl peroxide gel and 4\% Nicotinamide gel in mild to moderate acne of inflammatory origin.

\section{METHODS}

After obtaining clearance from the Institutional ethical committee and informed consent of all subjects, a prospective randomised open labelled comparative study with topical 5\% Benzoyl peroxide vs $4 \%$ Nicotinamide gel was conducted in Department of Dermatology, Dr B R Ambedkar medical college and hospital for a period of 6 months from June 2016 to December 2016.

A total of 60 subjects with mild to moderate acne was selected randomly at a ratio of $1: 1$. Thirty subjects belonging to Group I were treated with topical $5 \%$ Benzoyl peroxide twice daily for 4 weeks, another thirty subjects belonging to Group II were treated with $4 \%$ Nicotinamide gel twice daily for 4 weeks.

For each patient at baseline, details of sociodemographic data, history of acne, acne lesion counts and grade of acne was entered in the study proforma. Acne lesions were counted by the dermatologist. Inflammatory acne lesions were included in the study. Thus, total acne lesion count in each patient was considered as $100 \%$ baseline. Baseline clinical grading of acne severity was done according to Acne Global Severity Scale.

\section{Inclusion criteria}

Patients aged between 18 to 29 years; grade I-II acne; with written informed consent.

\section{Exclusion criteria}

Patients who were already on topical treatment for 2 weeks or systemic treatment with 4 weeks with either isotretinoin, hormones or antibiotics; those who had Nodulocystic acne, with facial dermatosis which interferes with evaluation in the study; patients suffering from immunocompromised states like Diabetes mellitus, AIDS; pregnancy and lactating mothers; those who had already received topical topical anti acne treatment (Benzoyl peroxide, retinoids, azelaic acid) in the last 1 month, also drugs producing acneiform eruptions either oral or topical drugs like steroids, Vitamin B12, anticonvulsive drugs.

Treatment regimen was under the direction of treating dermatologist. Patients were advised to wash the face and dry it well before application. 1 finger tip unit (approximately $0.5 \mathrm{gram}$ ) of each study drug was applied at bed time by dotting it over forehead, cheeks, chin and nose. A thin film was spread evenly over entire face avoiding periorbital, paranasal and perioral areas. Group I was treated with 5\% Benzoyl peroxide gel, Group II were treated with $4 \%$ Nicotinamide gel. Initially if there was irritation with the drugs they were advised to begin with short contact time of 15-30 minutes then gradually increase over upto 24 hours.

Patients were followed at the end of $2^{\text {nd }}$ week and $4^{\text {th }}$ week for efficacy and safety evaluation. At each follow up compliance was assessed verbally.

Efficacy assessment was done by spot counting of number of inflammatory acne lesions at follow ups with their respective basal counts. If any reduction was noted ,percentage reduction in acne lesion counts were calculated and expressed in terms of improvement in cne and graded.

Safety and tolerability was assessed by noting the local dryness, erythema, pruritus, hyperpigmentation, exfoliation, photosensitivity.

The data are expressed as mean, standard deviation for continuous measurements and number for categorical measurements. To find the significance of study parameters on continuous scale between two groups student's t test was used and for data on categorical scale Chi- square/ Fischer's extract test was used. The level of significance was taken as $\mathrm{p} \leq 0.05$ - significant, $\mathrm{p} \leq 0.001$ highly significant and $\mathrm{p} \geq 0.05$ - not significant.

\section{RESULTS}

Table 1: Demographic and baseline characteristics of acne patients studied in two groups.

\begin{tabular}{|c|c|c|c|}
\hline Characteristics & Group I & Group II & P value \\
\hline \multicolumn{4}{|l|}{ Age in years } \\
\hline $18-24$ & 70 & 86.7 & \multirow{3}{*}{0.515} \\
\hline $25-29$ & 30 & 13.3 & \\
\hline Mean \pm SD & $22.30 \pm 2.63$ & $21.90 \pm 2.07$ & \\
\hline \multicolumn{3}{|l|}{$\operatorname{Sex}(\%)$} & \multirow{3}{*}{0.598} \\
\hline Female & 56.7 & 63.3 & \\
\hline Male & 43.3 & 36.7 & \\
\hline \multicolumn{4}{|c|}{ Number of lesions distribution-Before treatment } \\
\hline $1-5$ & 0 & 0 & \\
\hline $6-10$ & 6.7 & 3.3 & \\
\hline $11-15$ & 50 & 53.3 & \\
\hline $16-20$ & 43.3 & 43.3 & \\
\hline
\end{tabular}

The study enrolled 60 patients with 30 patients in Group I and 30 patients in Group II. Both the treatment groups were comparable for their demographic characteristics and baseline disease characteristics. Common age group involved in the study was 18-24 years with predominantly females $56.7 \%$ in Group I and $63.3 \%$ in Group II 
respectively. The number of lesions counted were from 1 to 20 among them maximum was found between 11-15 lesions with $50 \%$ and $53.3 \%$ in Group I and Group II respectively (Table 1 ).

Patients were analysed in two grades - Grade 1 with lesions of acne counted from 1-10, Grade II with lesions counted from 11-20. It was noticed that maximum patients presented with lesions from 11-20 belonged to grade II (Table 2).

Table 2: Distribution of patients of acne based on grade of acne.

\begin{tabular}{|lll|}
\hline Grade of Acne & Group I & Group II \\
\hline Grade I & $2(6.7 \%)$ & $1(3.4 \%)$ \\
\hline Grade II & $28(93.3 \%)$ & $29(96.6 \%)$ \\
\hline
\end{tabular}

Patients aged from 18-29 years were included in the study. Majority of patients were between 18-24 years i.e. $70 \%$ and $86.7 \%$ in Group I and Group II respectively (Figure $1)$.

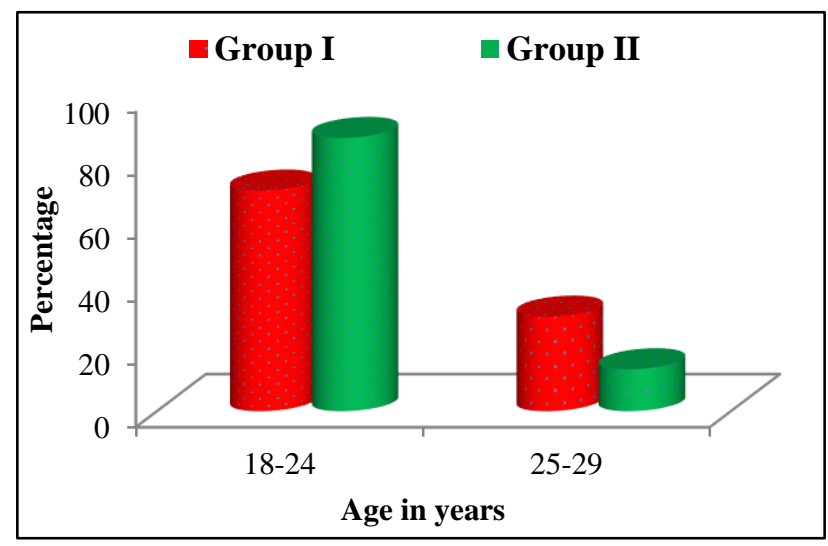

Figure 1: Distribution of acne based on age of the patient.

Both males and females were included in this study among them females were found to have a greater occurrence of acne i.e. $57 \%$ and $63 \%$ in Group I and Group II respectively (Figure 2).

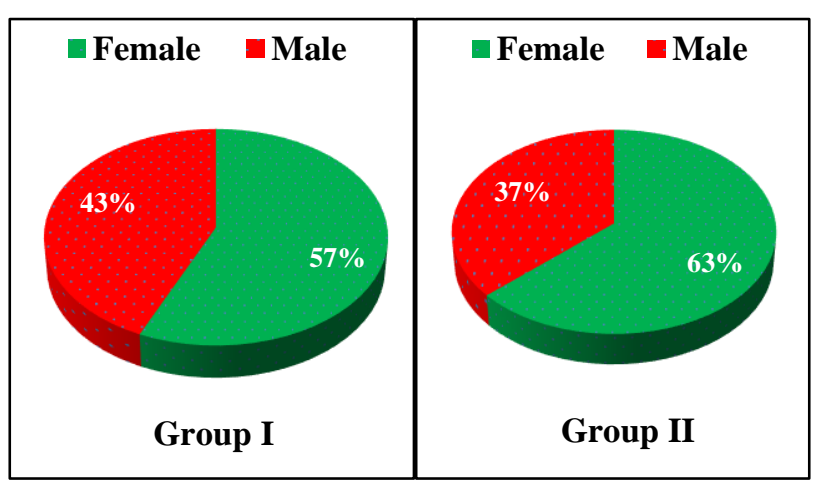

Figure 2: Indicates the gender distribution between the two groups.
Patients were treated with $5 \%$ Benzoyl peroxide for Group I and $4 \%$ Nicotinamide gel for Group II respectively. Percentage of reduction of number of lesions were compared in both the groups which showed percentage reduction of $32.3 \%$ in group I compared to group II of $22.37 \%$ at the end of 2 weeks with a p value of 0.001 which was statistically significant. At the end of 4 weeks there was a percentage reduction of $58.03 \%$ in Group I as compared to $36.07 \%$ in group II which showed $p$ value as $<0.001$ which was statistically significant (Table 3 , Figure $3)$.

Table 3: Percentage of reduction in number of lesions.

\begin{tabular}{|llll|}
\hline $\begin{array}{l}\text { Percentage of } \\
\text { reduction in number } \\
\text { of lesions }\end{array}$ & $\begin{array}{l}\text { Group } \\
\text { I }\end{array}$ & $\begin{array}{l}\text { Group } \\
\text { II }\end{array}$ & P value \\
\hline At 2 weeks & 32.3 & 22.37 & 0.001 \\
\hline At 4 weeks & 58.03 & 36.07 & $<0.001$ \\
\hline & & \multicolumn{2}{l|}{ Student t test } \\
\hline
\end{tabular}

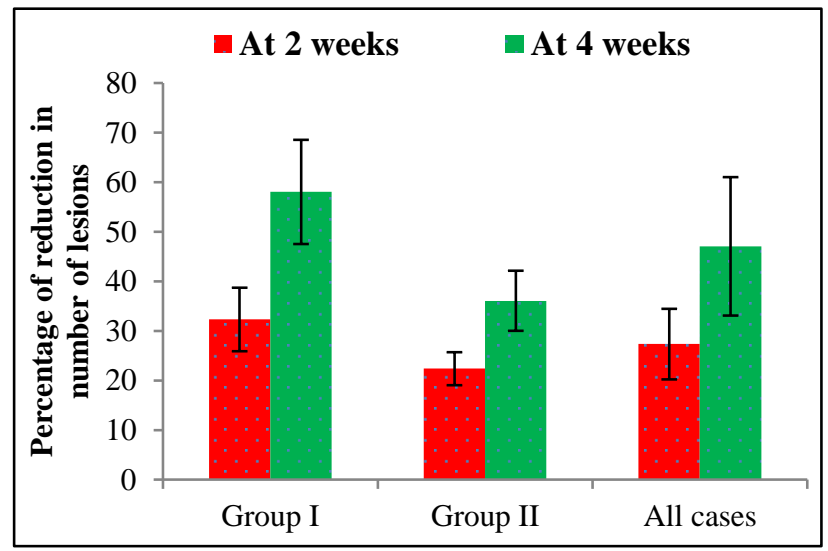

Figure 3: Indicates percentage of reduction in number of lesions at the end of 2 weeks and 4 weeks.

\section{DISCUSSION}

Acne vulgaris is an inflammatory disorder of pilosebaceous unit which occurs as a result of follicular epidermal hyperproliferation, increased sebum production, microbial flora changes and inflammation. The influence of androgen, P. acnes infection, some medications, genetic causes and stress are the main factors responsible for acne development. ${ }^{6}$ As far as treatment is concerned, topical therapy is the standard treatment for mild to moderate acne vulgaris. ${ }^{7}$

Benzoyl peroxide and antibiotics are the topical antimicrobials which have efficacy in treating inflammatory acne. ${ }^{8}$

Topical niacinamide has marked anti-inflammatory properties. $^{9}$

This study was undertaken to compare the antiinflammatory efficacy of $5 \%$ benzoyl peroxide gel versus 
4\% Nicotinamide gel for the topical treatment of mild to moderate acne vulgaris. In this study more acne patients were belonging to $18-24$ years (group I $-70 \%$ and group II $-86.7 \%$ ) with female preponderance $56.7 \%$ and $63.3 \%$ in group I and group II respectively. Similar age distribution and female preponderance was seen in a study done by Rallis E et al. ${ }^{10}$

Group I treated with 5\% Benzoyl peroxide were followed up at 2 weeks and 4 weeks which showed $32.3 \%$ and $58.03 \%$ percentage reduction of number of lesions respectively. This data correlates with the study conducted by Mills $\mathrm{OH}$ et al, in 1986, who demonstrated $40.3 \%$ and $55.9 \%$ percentage of reduction in acne lesions from baseline at the end of 4 weeks and 8 weeks respectively which is in concordance with our study. ${ }^{11}$

Group II were treated with $4 \%$ Nicotinamide gel and were followed up at 2 weeks and 4 weeks which showed $22.37 \%$ and $36.07 \%$ percentage of reduction in number of lesions which is similar with the study done by Shalita A et al, who demonstrated $60 \%$ reduction in the number of lesions at the end of 4 weeks treated twice daily, whereas in this study we treated the patient once daily at bed time. ${ }^{12}$

In this study, the effects of $5 \%$ benzoyl peroxide was compared with $4 \%$ nicotinamide gel in the topical treatment of mild to moderate acne vulgaris and it was found that there was a statistical significant difference ( $p$ $\leq 0.001)$ in efficacy between groups I (5\% benzoyl peroxide gel) and group II (nicotinamide gel) which implies benzoyl peroxide has better efficacy as compared with $4 \%$ Nicotinamide gel.

Finally to conclude topical 5\% Benzoyl peroxide has better efficacy compared to $4 \%$ Nicotinamide gel in the treatment of inflammatory mild to moderate acne.

\section{ACKNOWLEDGEMENTS}

Authors would like to thanks to the faculty and staff of department of Dermatology for their support in this study.

Funding: No funding sources Conflict of interest: None declared

Ethical approval: The study was approved by the Institutional Ethics Committee

\section{REFERENCES}

1. Katsambas AD, Stefanaki C, Cunliffe WJ. Guidelines for treating acne. Clin Dermatol. 2004;22:439-44. PMID: 15556732.

2. Healy E, Simpson N. Acne vulgaris. BMJ. 1994;308:831-3. PMID: 8167492

3. White GM. Acne therapy. In: James WD, Cockerell CJ, Dzubow LW, Paller AS, Yancey KB. Advances in dermatology. St Louis: Mosby; 1999:29-59.

4. Bissett DL, Oblong JE, Berge CA. Niacinamide: A B Vitamin that improves aging facial skin appearance. Dermatol Surg. 2005;31:860-5. PMID: 16029679

5. Draelos ZA, Matsubara A, Smiles K. The effect of $2 \%$ niacinamide on facial sebum production. J Cosmet Laser Ther. 2006;8:96-101. PMID: 16766489

6. Ray C, Trivedi P, Sharma V. Acne and its treatment lines. Int J Res Pharma Bio. 2013;3:1-16.

7. Rathi SK. Acne vulgaris treatment: The current scenario. Indian J Dermatol. 2011;56:7-13.

8. Kraft J, Freiman A. Management of acne. CMAJ. 2011;183:E430-435.

9. Wozniacka A, Sysa-Jedrzejowska A, Adamus J, Gebicki J. Topical application of NADH for the treatment of rosacea and contact dermatitis. Clin Exp Dermatol. 2003;28:61-3. PMID: 12558633

10. Rallis E, Verros C, Katoulis A, Katsarou A. Topical 5\% Benzoyl peroxide and 3\% Erythromycin gel: Experience with 191 patients with papulopustular acne. Acta Dermatovenerol Croat. 2013;21(3):160-7.

11. Mills $\mathrm{OH}$, Kligman AM, Pochi P, Comite H. Comparing $2.5 \%, 5 \%$, and $10 \%$ benzoyl peroxide on inflammatory acne vulgaris. International Journal of Dermatology. 1986 Dec 1;25(10):664-7.

12. Shalita AR, Smith JG, Parish LC, Sofman MS, Chalker DK. Topical nicotinamide compared with clindamycin gel in the treatment of inelammatory acne vulgaris. International Journal of Dermatology. 1995 Jun 1;34(6):434-7. PMID: 7657446

Cite this article as: Kumar ASN, Madan Mohan NT, Shwetha H, Shanmukananda P, Veena DR, Dube U. A prospective randomised open labelled comparative study of anti inflammatory effects of topical $5 \%$ benzoyl peroxide gel vs topical $4 \%$ nicotinamide gel for grade I-II acne in a tertiary care hospital. Int J Basic Clin Pharmacol 2018;7:952-5. 